\title{
Antibiotic susceptibility diagnostics for the future
}

\author{
With drug resistance on the rise, improvements in clinical antibiotic susceptibility testing and investment in \\ widespread implementation are needed to usher in a new generation of diagnostics that can inform on diverse \\ types of drug resistance and quickly predict drug susceptibility with high accuracy.
}

A ntibiotic resistant bacteria are a growing problem, and the spread of resistance means that antibiotic susceptibility cannot easily be predicted based on bacterial species alone. Thus, it is increasingly important to specifically test for resistance to shorten treatment times, ensure efficacy and reduce the likelihood of selecting for drug-resistant pathogens.

Current antibiotic susceptibility testing (AST) relies largely on time-consuming culture-based methods. After pathogens are initially grown from patient samples, a pure culture is challenged with different antibiotics and bacterial growth is assessed, either on agar, by measuring zones of inhibition around a disk or strip impregnated with antibiotic or by assessing turbidity in liquid media containing a drug. In both approaches, the minimum inhibitory concentration (MIC) of a panel of antibiotics is determined, and these values are compared against recommended resistance breakpoints, which are derived from the pharmacokinetic and pharmacodynamic profiles of the drugs for particular pathogens, as well as epidemiological data comparing MICs to treatment efficacy.

Culture-based AST has several limitations. First, it is time-consuming, taking days to culture pathogens and then test for susceptibility, during which time clinicians have likely already initiated empirical and potentially suboptimal antibiotics. Second, culturing is complicated - despite standardization of Mueller-Hinton media for AST, different recipe modifications are needed to support fastidious pathogens, ensure reproducibility for certain drugs (for example, cation concentrations affect polymyxin activity) or even activate resistance genes (for example, erythromycin pretreatment to induce clindamycin resistance). Third, clinically relevant resistance breakpoints can be difficult to define if we lack clear associations between MICs and successful outcomes (for example, resistance to newly approved drugs is rarely tested).

Also, the use of MICs and zones of inhibition have insufficient resolution to identify emerging resistance phenomena that have been implicated in clinical treatment failures, including persistence (the ability of some bacteria to tolerate, but not grow in, the presence of drug) or heteroresistance (where a subset of bacteria can replicate in antibiotic despite the majority of the population being susceptible). Recent studies report that many clinical strains are heteroresistant against different antibiotics. Andersson and colleagues (http://doi.org/c96t) found that a subset of cells can spontaneously and unstably amplify genes that promote drug resistance; and in this issue, Weiss and colleagues (http://doi.org/c96v) find that current AST approaches are not sensitive enough to capture this population heterogeneity, and often misclassify heteroresistant strains as either drug susceptible or fully resistant. In the former case, one could end up treating with an ineffective drug, and in the latter, potentially useful drugs might be ruled out. Indeed, the study showed that combining drugs for which strains are heteroresistant can successfully treat infections in mice. In these studies, heteroresistance was assessed through dilution and plating for colony-forming units, which would be laborious and difficult to routinely perform in the clinic. Thus, AST methods that have better sensitivity to analyse heterogeneous population responses are needed to capture different resistance phenomena.

The use of genomic sequencing for AST is promising and may speed up diagnosis by bypassing culturing on antibiotic. Sequencebased tests (usually based on PCR or hybridization) are already used in the clinic for example, detection of rifampin resistanceconferring mutations in Mycobacterium tuberculosis. The major limitation of using sequencing is having phenotypic validation that particular genomic markers are predictive, as the impact of certain mutations or known resistance genes can vary across organisms, and new polymorphisms or genes can drive unexpected drug resistance. Case in point is the work from Harrison and co-workers in this issue (http://doi.org/ c96w), which showed that many methicillinresistant Staphylococcus aureus strains have evolved changes in a gene conferring $\beta$-lactam antibiotic resistance that increases strain susceptibility to a combination of the $\beta$-lactam penicillin and a $\beta$-lactamase inhibitor. One might have predicted full resistance to all $\beta$-lactam drugs, given the detection of the resistance gene. Thus, there is still a need for phenotypic testing to refine sequencebased prediction and to capture cases where resistance arises without identifiable genomic changes. Automated dispensing and plates with pre-loaded drugs have increased the throughput of broth-based MIC detection in clinical labs, and non-turbidometric technologies are promising for even quicker and more sensitive phenotypic AST. These include the use of mass spectrometry to detect evidence of microbial activity or replication and microfluidic-based single cell imaging to quickly see changes in bacterial morphology in the presence of antibiotic.

However, developing new AST methods alone is not sufficient, as an underappreciated difficulty in diagnostic implementation is pressure from hospital budgets. Given the number of patients and samples to test, optimal tests are those that can maximize the number of useful diagnoses for as many patients as possible, while minimizing cost and labour. Some of the sequencing, mass spectrometry or imaging technologies require large investments in expensive machines and reagents, and involve specialized training for operators. On the other hand, if new AST diagnostics can outperform (or complement) current tests in key areas, there will likely be significant offsets in costs associated with hospitalization time, number of medical procedures performed and general increases in community health. Despite a seemingly large investment, the benefits of injecting money into the development of new (also, cheap and point-of-care) AST diagnostics are manifold.

Pathogens continue to acquire a diversity of mechanisms to counteract existing antibiotics, and clinicians are now challenged to find new therapeutic regimens to treat highly-resistant strains, including those that encode cryptic antibiotic resistance and tolerance mechanisms that our current tests can't detect. As the adage goes, necessity is the mother of invention, and before antibiotic resistance becomes universal, we have an opportunity to invest in the next generation of AST diagnostics that can provide faster, more reliable and more informative results. We must not waste it.

Published online: 20 September 2019 https://doi.org/10.1038/s41564-019-0577-4 